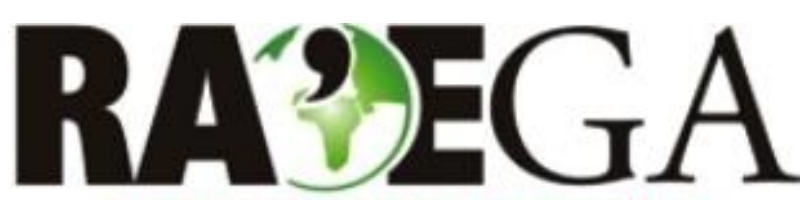

O ESPACYO GEOGRÁFICO EM ANÁLISE

\title{
RUÍDO OCUPACIONAL EM ESTAÇÕES DE ÔNIBUS - ESTUDO DE CASO EM ESTAÇÕES TUBO - CURITIBA - PARANÁ - BRASIL
}

\section{OCCUPATIONAL NOISE IN BUS STATIONS - CASE STUDY IN TUBE STATIONS - CURITIBA - PARANÁ - BRAZIL}

\author{
David Queiroz Sant'ana \\ Universidade Federal do Paraná \\ Curitiba, PR, Brasil \\ e-mail:davidqueirozsantana@gmail.com \\ Paulo Henrique Zannin \\ Universidade Federal do Paraná \\ Curitiba, PR, Brasil \\ e-mail:paulo.zannin@gmail.com
}

Recebido em: 05/05/2015

Aceito em: 20/05/2016

\section{Resumo}

O presente trabalho teve por objetivo investigar o nível de ruído ao qual o cobrador das estações de embarque e desembarque de passageiros das linhas expressas do sistema de transporte da cidade de Curitiba se encontra exposto durante sua jornada de trabalho. Toma-se para análise as estações-tubo localizadas nas avenidas Pe. Anchieta e Sete de Setembro, trechos de maior adensamento habitacional e de maior renda média da região sul da cidade. As altas densidades e o perfil econômico produzem um maior fluxo de veículos leves o que colabora para o aumento dos níveis de ruído. De um total de 9 estações em que foram efetuadas medições do nível equivalente de ruído (Leq) em intervalos de 10 minutos, observa-se conformidade acústica do ambiente de trabalho nas estações, quando consideradas as recomendações da norma nacional NR - 15 a qual legisla sobre o ruído ocupacional. Nas 9 amostras estudadas os níveis sonoros equivalentes medidos tem valores inferiores a 80 $\mathrm{dB}(\mathrm{A})$, nível sonoro que a partir do qual há a indicação de risco auditivo. Entretanto, sob as prescrições da norma brasileira que estabelece os padrões de ergonomia para 0 ambiente de trabalho NR - 17 esses ambientes caracterizam-se como desconfortáveis na totalidade das amostras, pois registram níveis sonoros equivalentes acima de $L e q=65 \mathrm{~dB}(\mathrm{~A})$.

Palavras-chave: Ruído; Ruído Ocupacional; Poluição Sonora; Medições do ruído; Legislação. 


\begin{abstract}
This study investigates the level of noise to which bus fare collectors in Curitiba's express bus stations are exposed during their workday. The object of analysis are the tube bus stations located on Padre Anchieta and Sete de Setembro avenues in the south sector of the city of Curitiba, Brazil, which is characterized by its high housing density and high average per capita income. The high densities and economic profile produce a greater flow of light vehicles which contributes to the increase in noise levels. At the 9 bus stations, where equivalent noise levels (Leq) were measured, at 10-minute intervals, the workplace noise levels, were found to be in compliance with the limits established by the Brazilian national standard NR-15, which address occupational noise exposure, since the 9 samples studied here, showed equivalent noise levels below $80 \mathrm{~dB}(\mathrm{~A})$, sound level from which there is an indication of auditory risk. However, the environments of all the samples showed measured equivalent sound levels exceeding Leq $=65 \mathrm{~dB}(\mathrm{~A})$, thus characterizing them as uncomfortable according to Brazil's regulatory standard NR-17, which establishes ergonomic standards for the workplace.
\end{abstract}

Keywords: Noise; Occupational noise; Noise pollution; Noise measurement; Legislation.

\title{
1. INTRODUÇÃO
}

Desde a Antiguidade, mais precisamente na época do Império Romano (50 - 44 a.C.) o ruído já causava incômodo à população. Júlio César, sendo então governador naquela época, decretou a proibição de fazer rodar, durante a noite, os pesados veículos movidos à tração animal sobre pavimento de pedra, para não perturbar o sono da população (NUDELMANN et. al., 1997).

Atualmente, o desenvolvimento científico e a evolução tecnológica aumentaram os níveis de ruído ocupacional, ruído urbano (proveniente principalmente de veículos de transporte) e o ruído decorrente de atividades de lazer (academias de ginástica, academias de tiro, brinquedos e outros) e como consequência, trouxeram prejuízos para o bem estar da população que está frequentemente exposta a este agente físico (FIORINI, 2009).

A poluição sonora é atualmente, segundo a Organização Mundial da Saúde (WHO, 2003) uma das principais formas de poluição no ambiente urbano e no ambiente de trabalho. Ela é responsável por impactos negativos com prejuízos ao meio ambiente e a qualidade de vida da população. A 
Organização Mundial da Saúde (World Health Organization - WHO (2011) classifica a poluição sonora como o terceiro tipo de poluição que mais atinge a população mundial, depois da poluição do ar por emissões gasosas e da poluição da água. A WHO também considerada a poluição sonora como um caso de saúde pública. A WHO (2011) enfatiza ainda que o crescimento da poluição sonora é insustentável, pois afeta diretamente a saúde, causando aborrecimentos, alterações comportamentais, estresse relacionado aos efeitos psicológicos, interferência na comunicação, além de atrapalhar o repouso, o relaxamento e o sono.

O ruído é hoje também classificado como um tipo de poluição tóxica. Ele aparece junto a outros problemas de poluição ambiental como: o aquecimento global, a destruição da camada de ozônio, dentre outros. A Tabela 1 adaptada de Santos de Souza (2000), mostra resumidamente os principais problemas ambientais, sua abrangência e os principais agentes geradores de poluição.

Tabela 1 - Principais problemas de poluição ambiental

\begin{tabular}{|c|c|c|}
\hline $\begin{array}{c}\text { Problema } \\
\text { Ambiental }\end{array}$ & Abrangência & \multicolumn{1}{c|}{ Principal Poluidor } \\
\hline $\begin{array}{c}\text { Efeito Estufa e } \\
\text { mudanças climáticas }\end{array}$ & Global & $\begin{array}{c}\text { Emissão de } \mathrm{CO}_{2} \text {, metano e } \\
\text { desmatamento. }\end{array}$ \\
\hline \hline $\begin{array}{c}\text { Destruição da } \\
\text { camada } \\
\text { de ozônio }\end{array}$ & Global & Emissão de clorofluorcarbonos \\
\hline \hline Poluição tóxica & Continental & $\begin{array}{l}\text { Emissão de } \mathrm{SO}_{2}, \text { particulados, } \\
\text { metais pesados, agroquímicos, } \\
\text { ruído }\end{array}$ \\
\hline
\end{tabular}

Fonte: Adaptado de Ekins, P. e Jacobs, M.: "Environmental Sustentability and the Growth of GDP: Conditions for Compatibility. World Institute for Development Economics Research (WIDER) conference at Oxford, June 30 - July 2, 1993". 
Em pesquisa realizada na cidade de Curitiba (ZANNIN et.al., 2003), foi identificado que dentre as fontes de ruído, o tráfego de veículos foi considerado como a fonte sonora mais incômoda para a maioria dos entrevistados $(73 \%$ das respostas).

Reconhecida internacionalmente por sua experiência em planejamento urbano e de tráfego, Curitiba possui um sistema de transporte coletivo baseado em ônibus de grande capacidade de passageiros. Estes ônibus trafegam em pistas exclusivas que compreendem aproximadamente $72 \mathrm{~km}$ de extensão.

O sistema de transporte urbano atende a aproximadamente 1.900 .000 passageiros diariamente (ZANNIN, 2006). O sistema de transporte urbano de Curitiba tem sido alvo de diversos estudos a nível internacional (HERBST, 1992; SMITH e HENSHER, 1998; DIESENDORF, 2000). Os grandes corredores de circulação de ônibus em vias exclusivas compõem os eixos hierarquizadores da malha viária urbana ordenando os eixos de desenvolvimento da cidade de Curitiba (FIEDLER e ZANNIN, 2015).

Os eixos de circulação constituem-se de três vias: duas marginais de fluxo rápido e uma central de fluxo lento conhecidos como sistema trinário. Na via central, os ônibus rodam em uma pista exclusiva ao centro, enquanto os demais veículos utilizam as duas pistas laterais (ver Figura 1):

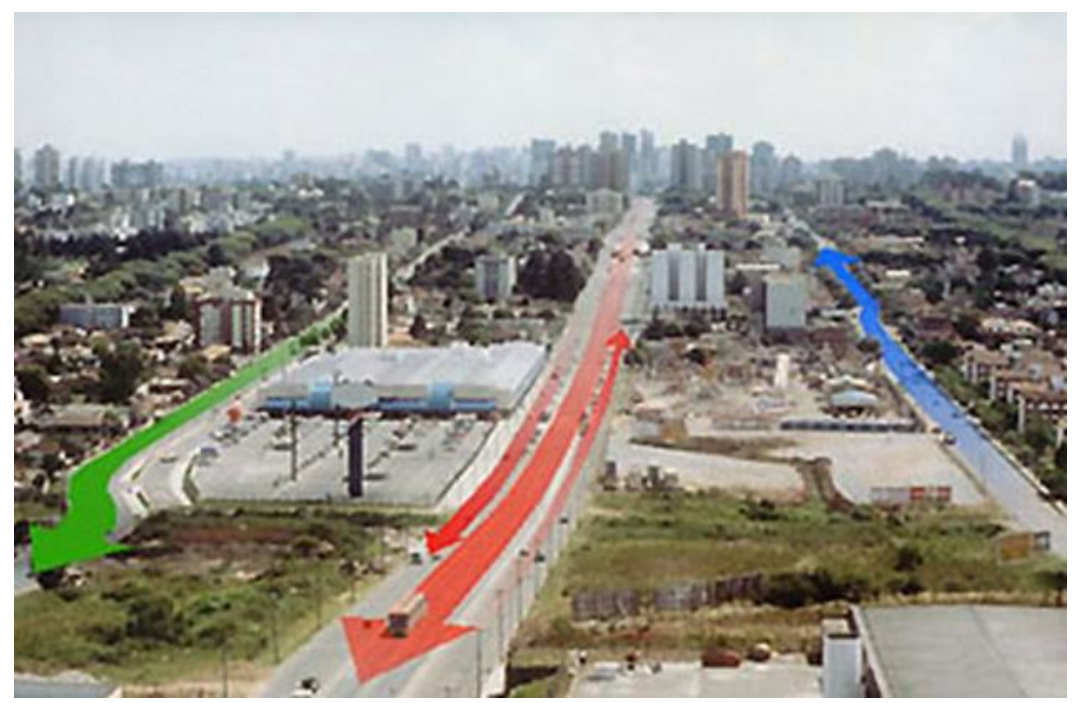

Figura 1: Sitema Trinário: Mostra a via central exclusiva para o transporte coletivo, e duas vias paralelas de sentidos opostos para a circulação dos veículos. Fonte: IPPUC. 
Os ônibus que percorrem os eixos de transporte nas vias exclusivas do sistema trinário, são de modelos diferentes dos veículos que trafegam junto aos automóveis. São veículos mais longos, compostos por três seções integradas por uma conexão que permite a rotação e a execução das curvas, e apresenta fechamento externo sanfonado de material emborrachado (ver Figura 2).

Os ônibus tipo bi-articulado apresentam as seguintes características técnicas:

- Comprimento: $24,52 \mathrm{~m}$

- Largura: 2,50m

- Altura: $3,415 \mathrm{~m}$

- Chassis e motorização Volvo;

- Motorização: 340cv diesel;

- Transmissão automática, 5 marchas;

- Suspensão a ar;

- Capacidade: 270 passageiros;

- Embarque: elevado através das estações tubo.



Figura 2: Ônibus tipo bi-articulado.

As estações-tubo consistem em plataformas de embarque e desembarque de passageiros, para a utilização dos ônibus tipo bi-articulado. 0 embarque da estação tubo se diferencia do convencional por apresentar uma 
diferença de nível em relação ao solo. Esta diferença de nível é de aproximadamente $60 \mathrm{~cm}$. O passageiro faz um embarque numa plataforma elevada, na qual um cobrador controla a entrada da estação, o que faz diminuir o tempo de embarque nos ônibus.

As estações-tubo permitem o acesso de pessoas portadoras de deficiência através de elevadores instalados em uma de suas extremidades. As estações-tubo totalizam 351 unidades por toda a cidade e apresentam as seguintes características:

- Estrutura tubular em aço;

- Vedação em vidro;

- Capacidade de aproximadamente 50 pessoas.

- Elevador para pessoa portadora de deficiência.

- Capacidade: 45 pessoas.

A Figura 3 apresenta um modelo de estação tubo:

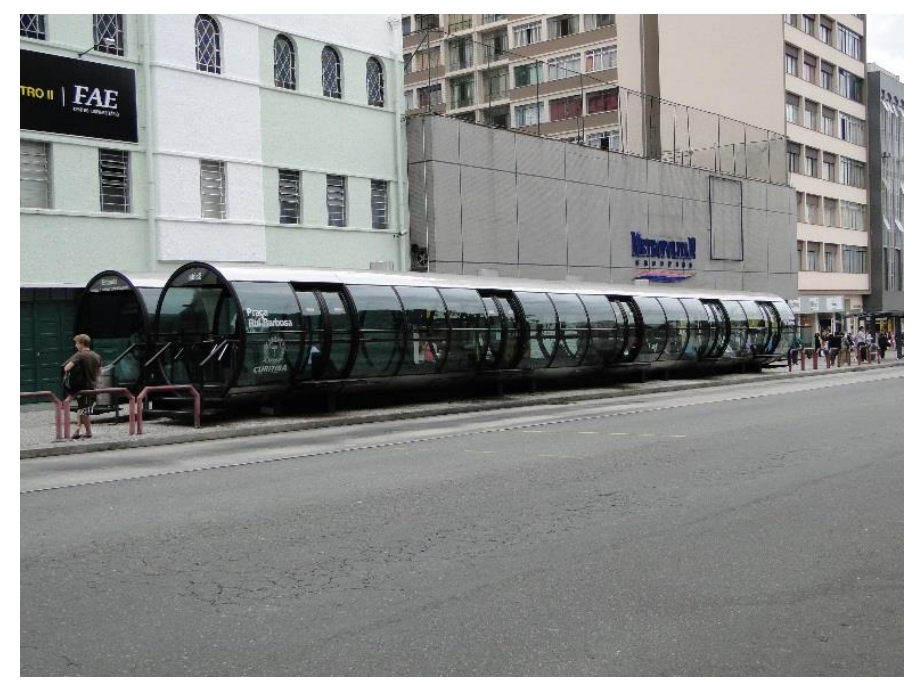

Figura 3: Estação Tubo.

Estudos têm abordado a problemática do ruído ambiental englobando, por exemplo, áreas educacionais (HÉTU et al, 1990; KENNEDY et al, 2005; THAKUR, 2006; ZANNIN \& MARCON, 2007; ASTOLFI \& PELLERY, 2008; ZANNIN \& ZWIRTES, 2009; GOSWAMI, 2011). O ruído no aprendizado tem influência no comportamento e na compreensão dos estudantes, sendo que 
locais muito ruidosos são desfavoráveis para o aprendizado e tornam o ensino exaustivo (HAGEN et al, 2002).

O ruído no ambiente de trabalho, mais precisamente, o ruído no transporte público de Curitiba, foi avaliado em relação aos motoristas, no trabalho de PORTELLA \& ZANNIN (2008), e seus efeitos sobre os motoristas, no trabalho de PORTELLA et al (2013).

Segundo MASCHKE (1999), o organismo humano sofre dois tipos de dano quando exposto ao ruído. O primeiro específico, é o dano auditivo gerado pelo desgaste mecânico do tímpano, e o segundo, não específico corresponde às reações de stress, correspondentes aos distúrbios hormonais causadores de alterações nas funções orgânicas do indivíduo.

Em sua dissertação de mestrado, SANTOS (2015) avaliou os níveis de ruído nas circunvizinhanças das estações tubo. Deve-se ressaltar que SANTOS (2015) não fez as medições dentro das estações-tubo. Seu objetivo foi mostrar se as estações-tubo, como elementos do meio ambiente urbano, se enquadravam dentro da Lei do Silêncio de Curitiba. Portanto, o foco da pesquisa, foi identificar se as estações tubo, estavam em conformidade com a Lei Ambiental 10.625 da cidade de Curitiba. Esta lei divide a cidade em diversas zonas urbanas, e estabelece para as mesmas, limites de ruído para os diversos períodos do dia (diurno, vespertino, noturno). Esta lei trata da avaliação do ruído ambiental e não do ruído no ambiente de trabalho.

O foco do presente trabalho, foi avaliar os níveis sonoros que atingem 0 cobrador dentro do seu ambiente de trabaho, ou seja, o indivíduo que fica dentro das estações tubo. Estes trabalhadores recebem o ruído do meio ambiente urbano (ruído de veículos, ruído de trens, ruído de aviões, sirenes, ruído da construção civil, etc). Eles recebem também o ruído proveniente dos ônibus que param nas estações tubo, para desembarcar e embarcar passageiros. Estas questões inserem-se dentro do problema do ruído ocupacional, e são avaliadas por Normas Regulamentadoras do Ministério do Trabalho, como a NR 15 e a NR 17, e pela norma internacional - Norma ISO 1999 (1990): Acoustics - Determination of occupational noise exposure." Também foi usada a norma NHO-01 da FUNDACENTRO. A norma NHO-01 
apresenta além de uma avaliação quantitativa do ruído, uma avaliação qualitativa do mesmo, conforme pode ser visto na Tabela 2.

O objetivo final do presente trabalho, foi fazer a comparação dos níveis sonoros medidos com os níveis sonoros limites estabelecidos pelas normas que avaliam a qualidade do ambiente de trabalho. Estas normas, como acima citadas, são as Normas Regulamentadoras NR 15 e NR 17 e a Norma de Higiene Ocupacional NHO-01. Uma vez feita esta comparação, foi possível classificar o ambiente de trabalho como salubre ou insalubre com relação ao agente físico ruído, ou seja, a poluição sonora, segundo a NR 15 e NHO-01 e confortável ou desconfortável segundo a NR 17, que trata da questão ergonômica dos ambientes de trabalho.

\section{MATERIAIS E MÉTODOS}

Para se realizar a avaliação do ambiente de trabalho dos cobradores, ou seja, dos profissionais que trabalham dentro das estações tubo, foram realizadas medições do nível sonoro equivalente Leq, expresso em $\mathrm{dB}(\mathrm{A})$. As medições foram realizadas com um medidor de nível de pressão sonora da marca Brüel \& Kjaer BK 2238.

Os procedimentos das medições, seguiram as indicações da norma internacional ISO 1999 (1990) - "Determination of occupational noise exposure". Esta norma indica que o medidor sonoro deve ser montado sobre um tripé, ficando o mesmo na altura do ouvido do indivíduo que está sendo avaliado. Ainda segundo a ISO 1999, a distância entre o ouvido do indivíduo avaliado e o microfone do medidor deve ser de $10 \mathrm{~cm}$.

Foram realizadas em cada estação tubo, três medições de 10 minutos cada uma. Posteriormente calculou-se a média logarítmica destas três medições, para cada estação tubo, e os resultados são apresentados nas Tabelas 3 e 4 . Foram avaliados os níveis sonoros em nove estações tubo. As medições foram realizadas entre os meses de março e abril, de segunda-feira até sexta-feira no período da tarde entre 13:30 e 16:30 hs. Foram analisadas as estações tubo do eixo da Rua Padre Anchieta, a saber: Estação Gastão 
Câmara, Estação Bigorrilho, Estação Bruno Filgueira e Estação Praça da Ucrânia. O outro eixo analisado foi o da Rua Sete de Setembro: Estação Bento Viana, Estação Coronel Dulcídio, Estação Praça Oswaldo Cruz e Estação Alferes Poli.

A Tabela 1 a seguir apresenta o tempo máximo de exposição diária permissível para ruídos contínuos ou intermitentes, conforme o Anexo 1 da Norma Regulamentadora NR 15 (NR-15, 2011, p.212).

TABELA 1: LIMITES DE TOLERÂNCIA PARA EXPOSIÇÃO AO RUÍDO NR - 15.

\begin{tabular}{|c|c|c|c|}
\hline $\begin{array}{c}\text { NÍVEL DE RUÍDO } \\
\text { Leq dB(A) }\end{array}$ & $\begin{array}{c}\text { MÁXIMA } \\
\text { EXPOSIÇÃO } \\
\text { DIÁRIA } \\
\text { PERMISSÍVEL }\end{array}$ & $\begin{array}{c}\text { NÍVEL DE RUÍDO } \\
\text { Leq } \mathrm{dB}(\mathrm{A})\end{array}$ & $\begin{array}{c}\text { MÁXIMA } \\
\text { EXPOSIÇÃO } \\
\text { DIÁRIA } \\
\text { PERMISSÍVEL }\end{array}$ \\
\hline 85 & 8 hours & 98 & 1 hour and 15 min \\
\hline 86 & 7 hours & 100 & 1 hour \\
\hline 87 & 6 hours & 102 & 45 minutes \\
\hline 88 & 5 hours & 104 & 35 minutes \\
\hline 89 & 4 hours and 30 min & 105 & 30 minutes \\
\hline 90 & 4 hours & 106 & 25 minutes \\
\hline 91 & 3 hours and 30 min & 108 & 15 minutes \\
\hline 92 & 3 hours & 110 & 10 minutes \\
\hline 93 & 2 hours and 40 min & 112 & 8 minutes \\
\hline 94 & 2 hours and 15 min & 114 & 7 minutes \\
\hline 95 & 2 hours & 115 & \\
\hline 96 & 1 hour and 45 min & & \\
\hline
\end{tabular}

Utilizou-se também, para qualificar o conforto no ambiente de trabalho dos cobradores, a Norma Regulamentadora NR 17. Esta norma estabelece, que ambientes de trabalho onde, o nível sonoro equivalente for superior a $65 \mathrm{~dB}(\mathrm{~A})$, são considerados desconfortáveis.

Também foi utilizada a Norma de Higiene Ocupacional - NHO-01 da FUNDACENTRO (1999). A NHO-01 estabelece uma avaliação quantitativa e uma avaliação qualitativa dos níveis sonoros medidos. A NHO-01 expressa níveis de ação que devem ser tomados, quando os níveis sonoros ultrapassarem valores limites de segurança a saúde do trabalhador. Os níveis 
sonoros limite, a consideração técnica e a atuação recomendada, segundo a NHO-01, estão listadas na Tabela 2:

TABELA 2: Níveis de ação conforme Norma de Higiene Ocupacional NHO-01

\begin{tabular}{|c|c|c|}
\hline $\begin{array}{c}\text { Leq } \\
\mathbf{d B}(\mathbf{A})\end{array}$ & $\begin{array}{c}\text { Consideração } \\
\text { Técnica }\end{array}$ & $\begin{array}{c}\text { Atuação } \\
\text { Recomendada }\end{array}$ \\
\hline \hline Até 82 & Aceitável & No mínimo manutenção da condição existente \\
\hline \hline 82 a 84 & Acima do nível de ação & $\begin{array}{c}\text { Adoção de medidas } \\
\text { preventivas }\end{array}$ \\
\hline \hline 84 a 85 & Região de incerteza & $\begin{array}{c}\text { Adoção de medidas } \\
\text { preventivas e corretivas } \\
\end{array}$ \\
& & visando a diminuição dos níveis sonoros \\
\hline \hline$>85$ & Acima do limite de & Adoção imediata de \\
& exposição & medidas corretivas \\
\hline
\end{tabular}

\subsection{Mapeamento Acústico}

Foi calculado neste trabalho o mapa de ruído, para duas estações tubo, a da Praça Oswaldo Cruz e da Rua Alferes Poli, que se encontram no eixo de estações tubo que apresentou os maiores valores de níveis sonoros. Como podemos observar das tabelas 3 e 4, o Eixo da Rua Sete de Setembro é o que apresenta os níveis sonoros mais elevados.

Um mapa de ruído é uma representação gráfica dos níveis de som de uma região. Os mapas de ruído podem ser utilizados para a avaliação dos níveis sonoros a curto ou longo prazo, de modo que eles possam ser utilizados para a pré-avaliação dos planos de ação e medidas de controle de ruído. Esse tipo de avaliação é muito útil para o planejamento urbano acústico, para o uso do solo, e para a avaliação de impacto ambiental sonoro de qualquer atividade antes, durante e após a sua implantação (ASENSIO et al, 2011). A utilização do mapeamento sonoro permite, além de uma melhor visualização do problema ocasionado pela poluição sonora, propor diferentes possíveis cenários, 
demonstrando o que pode ser feito para melhoria do problema. O SoundPLAN (1999) que foi o software utilizado neste trabalho é um software em ambiente MS-Windows ${ }^{\circledR}$, especialmente desenvolvido para o cálculo de mapas acústicos.

A primeira tarefa para as simulações acústicas consistiu em se fazer a modelagem das áreas a serem estudadas. Apesar do SoundPlan possuir um módulo para se modelar a região de estudo, é preferível fazer uso de um programa tipo CAD (Computer Aided Designer). Para tanto, o AutoCad versão $\mathrm{R} 14$, foi utilizado, e as áreas em estudo foram modeladas com a ajuda de plantas baixas. Depois de feito o desenho procedeu-se à colocação das edificações. Como os locais avaliados contém em sua grande maioria prédios, a altura de cada pavimento foi estimada em 3 metros, para a confecção do modelo. Após esta etapa chegou-se a finalização do modelo horizontal do Eixo da Rua Sete de Setembro com as estações tubo em análise. Na verdade, foram avaliadas as imediações de duas estações tubo, a da Praça Oswaldo Cruz e a da Rua Alferes Poli. Para a etapa final do modelo foi necessário acrescentar ainda os níveis sonoros equivalentes $L_{\text {Aeq }}$ medidos em cada estação tubo. Os mapas acústicos calculados são mostrados nas figuras 4 e 5 . Os softwares que calculam mapas acústicos são ainda ferramentas muito caras, mas apesar disto, sua utilização vem sendo aumentada largamente nos últimos anos (LEE et al, 2008; KING \& RICE, 2009; PINTO \& MARDONES, 2009; TSAl et al 2009; ZANNIN \& SANT'ANA, 2011; LAW et al, 2011; GUEDES et al, 2011; ZANNIN et al 2013; CASAS et al, 2014; BUNN, F. \& ZANNIN, P.H.T., 2016).

\section{RESULTADOS E DISCUSSÕES}

Os níveis medidos para o Eixo da Rua Padre Anchieta, níveis sonoros equivalentes entre $L_{\text {Aeq }} 74,4$ e $76 \mathrm{~dB}(A)$, e os níveis sonoros equivalentes entre 75,5 e 77,6 dB(A) para o Eixo da Rua Sete de Setembro, são apresentados nas Tabelas 3 e 4 . Portanto, percebe-se que os mesmos, estão em conformidade com a Norma Regulamentadora NR 15, pois os níveis sonoros medidos estão 
abaixo do limite de $L_{\text {Aeq }} 85 \mathrm{~dB}(\mathrm{~A})$, o que corresponde a uma jornada diária de 8 horas de trabalho.

Quando se aplica a norma Norma Regulamentadora NR 17, que trata da avaliação do conforto no ambiente de trabalho, percebe-se que todas as estações não atendem a norma NR 17. Para a NR 17, ambientes de trabalho, com níveis sonoros equivalentes acima de $65 \mathrm{~dB}(\mathrm{~A})$ são considerados desconfortáveis.

O uso de protetores auriculares para o cobrador não pode ser recomendado, pois o mesmo impediria o bom atendimento dos passageiros. Portanto, deve-se intervir na boa manutenção dos veículos em geral, e em particular dos ônibus que circulam nestas vias exclusivas, ou seja, os ônibus biarticulados. Deve-se também cuidar da manutenção das estações tubo como um todo. Em especial deve-se evitar os ruídos excessivos que são gerados, especialmente quando da abertura das portas e das plataformas de desembarque, e assim reduzir os níveis de ruído, e por conseguinte 0 desconforto dos cobradores e ou os possíveis danos auditivos ao longo dos anos de trabalho.

O uso da Norma de Higiene Ocupacional NHO-01, mostra que todos os níveis sonoros medidos estão abaixo de $82 \mathrm{~dB}(\mathrm{~A})$, portanto, a consideração técnica é - classificada como aceitável - e a atuação recomendada, é no mínimo a manutenção da condição existente - (ver Tabela 2).

TABELA 3: Nível sonoro equivalente $L_{\text {Aeq }}$ em dB, nas estações-tubo - Eixo da Rua Padre Anchieta.

\begin{tabular}{|c|c|}
\hline ESTAÇÃo TUBO & $\begin{array}{c}\text { NíVEL SONORO } \\
\text { EQUIVALENTE MÉDIO } \\
\text { Leq dB(A) }\end{array}$ \\
\hline \hline Gastão Câmara & 75,5 \\
\hline Bigorrilho & 74,5 \\
\hline \hline Bruno Filgueira & 75,6 \\
\hline \hline Praça da Ucrânia & 76 \\
\hline Presidente Taunay & 74,4 \\
\hline
\end{tabular}

TABELA 4: Nível sonoro equivalente $L_{\text {Aeq }}$ em dB(A), nas estações-tubo - Eixo da Rua Sete de Setembro. 
RUÍDO OCUPACIONAL EM ESTAÇÕES DE ÔNIBUS - ESTUDO DE CASO EM ESTAÇÕES TUBO - CURITIBA - PARANÁ - BRASIL

\begin{tabular}{|c|c|}
\hline ESTAÇÃO TUBO & NÍVEL SONORO \\
& EQUIVALENTE MÉDIO \\
Leq dB(A)
\end{tabular}

\begin{tabular}{|c|c|}
\hline Bento Viana & 77,6 \\
\hline \multicolumn{2}{|c|}{} \\
\hline Coronel Dulcídio & 75,5 \\
\hline \multicolumn{2}{|c|}{} \\
\hline Praça Oswaldo Cruz & 76,4 \\
\hline Alferes Poli & 76,5 \\
\hline
\end{tabular}

As figuras 4 e 5 apresentam os mapas acústicos para a via exclusiva de ônibus da Rua Sete de Setembro. A Figura 4 mostra o mapa acústico nas cercanias da Praça Oswaldo Cruz, e a Figura 5 mostra o mapa acústico nas cercanias da Rua Alferes Polis.

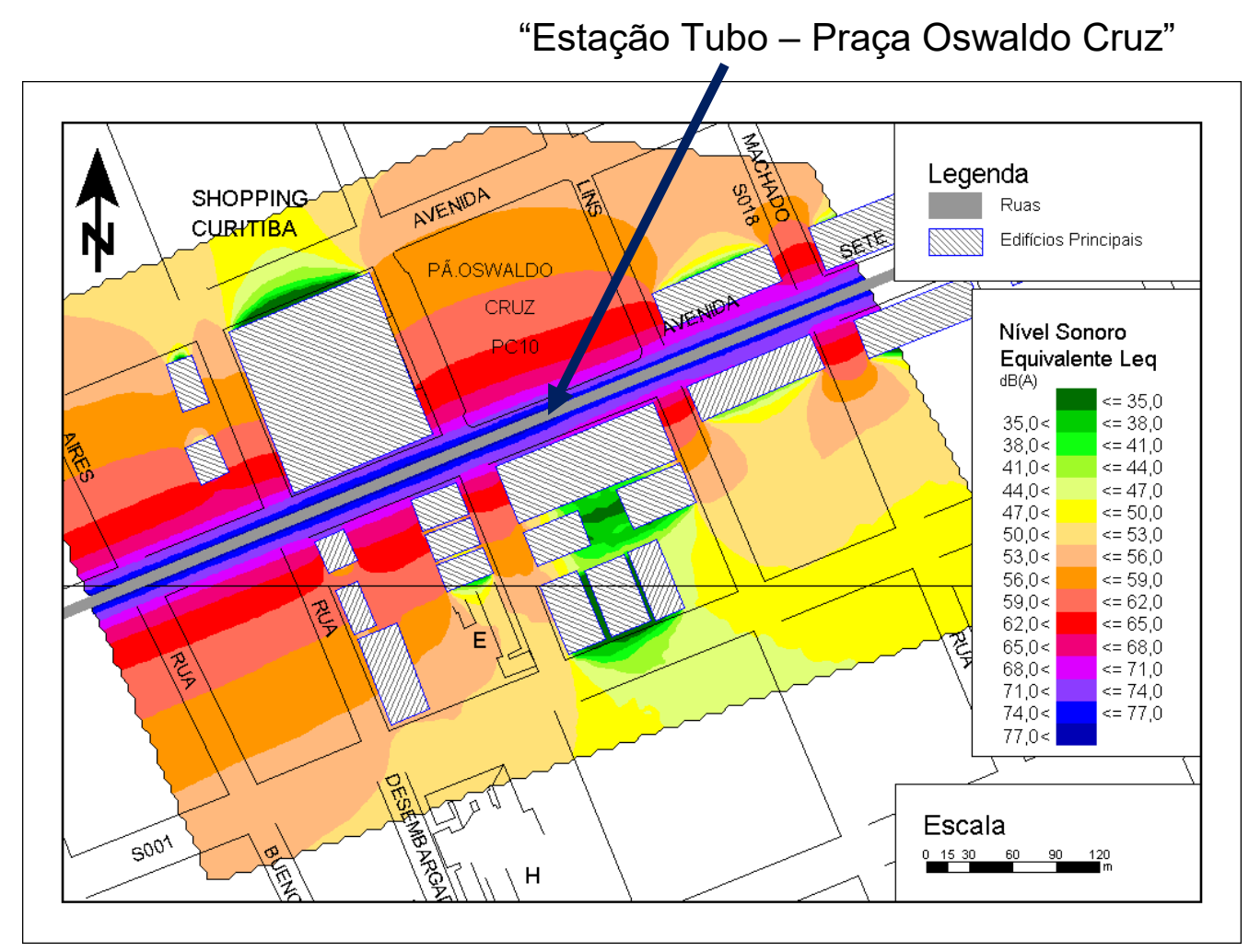

Figura 4 - Mapeamento acústico da via exclusiva de ônibus bi-articulado da Rua Sete de Setembro. Neste mapa pode-se observar a Praça Oswaldo Cruz, que dá nome a Estação Tubo Oswaldo Cruz, localizada no Eixo da Rua Sete de Setembro. 


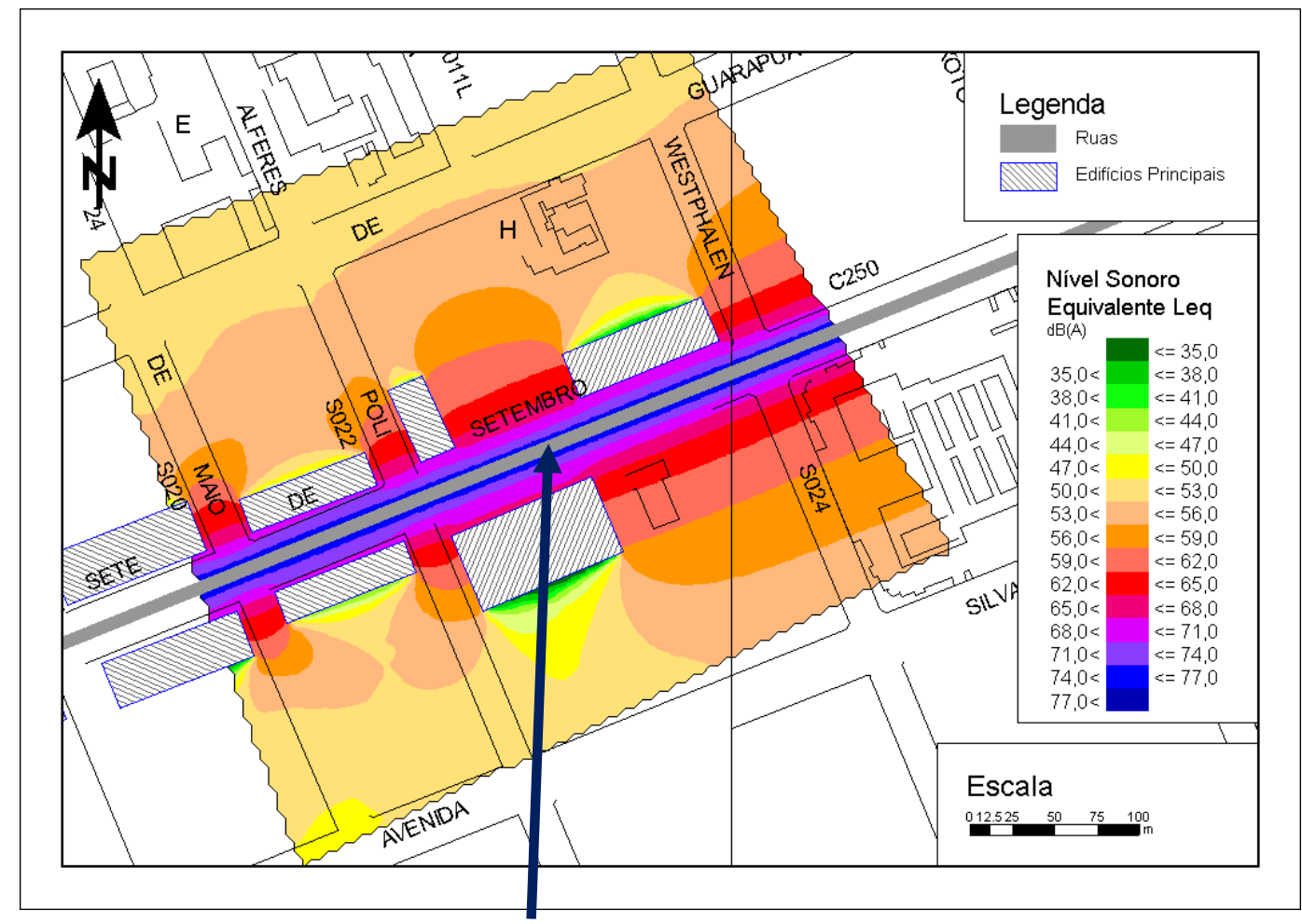

"Estação Tubo - Alferes Poli"

Figura 5 - Mapeamento acústico da via exclusiva de ônibus bi-articulado da Rua Sete de Setembro. Neste mapa pode-se observar a Rua Alferes Poli, que dá nome a Estação Tubo Alfares Poli, localizada no Eixo da Rua Sete de Setembro.

Observa-se das figuras 4 e 5 que os níveis sonoros no centro da via exclusiva para os ônibus bi-articulados, apresentam níveis sonoros variando entre 74 e $77 \mathrm{~dB}(\mathrm{~A})$, "cor azul". Estes valores compreendem os valores apresentados na Tabela 4, para a Praça Oswaldo Cruz e para a Rua Alferes Poli, nas quais os níveis sonoros medidos dentro das estações tubo foram respectivamente 76,4 e $76,5 \mathrm{~dB}(\mathrm{~A})$. Isto mostra como o ruído do ambiente atinge os cobradores dentro das estações tubo. Os mapas de ruído são uma excelente ferramenta para a avaliação do ruído, no entanto eles não substituem as medições. Como visto no exemplo aqui apresentado os valores do mapa calculado "cor azul" encampam os valores medidos de 76,4 e 76,5 dB(A). A avaliação do ruído com medições em campo e sua posterior simulação computacional são medidas complementares. Ambas são onerosas, pois os medidores sonoros são em sua grande maioria importados e necessita-se de 
mais de um medidor para varrer a avaliação de grandes áreas, assim como pessoal especialmente treinado para fazer estas medições. Por outro lado, o uso dos softwares de simulação para o cálculo dos mapas acústicos, necessitam de um número menor de profissionais envolvidos. No entanto estes profissionais devem possuir conhecimentos profundos das leis da acústica envolvidas no processo. Os softwares também são todos importados e ainda tem o agravante de serem muito caros, embora seu preço venha diminuindo.

\section{CONCLUSÕES}

Com base nos resultados medidos, e observações realizadas ao longo desta pesquisa, conclui-se que embora o ambiente de trabalho dos cobradores das estações-tubo esteja em conformidade com os parâmetros da Norma Regulamentadora NR-15 e em conformidade com parâmetros quantitativos e qualitativos da Norma de Higiene Ocupacional NHO-01, medidas de manutenção periódica preventivas das estações-tubo e dos ônibus biarticulados, que trabalham em conjunto com estas estações devem ser realizadas. Estas medidas ajudarão a garantir um ambiente de trabalho salutar aos cobradores que trabalham nas estações-tubo, pois o ruído é somente um dos problemas enfrentado por estes trabalhadores. Além do ruído, existe o problema da qualidade do ar e a questão da temperatura, tanto no verão como no inverno. Mas estas questões não são o foco do presente trabalho.

Todas as estações avaliadas mostraram-se desconfortáveis, quando se usa para a avaliação a Norma Regulamentadora 17 - NR 17 - a qual trata da questão ergonômica, ou seja, da questão do conforto dentro dos ambientes de trabalho. Esta norma indica, que ambientes que tenham níveis sonoros superiores a $65 \mathrm{~dB}(\mathrm{~A})$, são considerados desconfortáveis. Em todas as estações estudadas, os níveis sonoros médios foram maiores do que $65 \mathrm{~dB}(\mathrm{~A})$, conforme pode ser observado nas Tabelas 3 e 4.

Os mapas de ruído se apresentam como ferramentas importantes na análise do ruído em grandes áreas, pois possibilitam observar a sua distribuição espacial. Os mapas de ruído podem servir para se fazer predições 
acústicas e assim poupar tempo e dinheiro nas avaliações de impacto ambiental. As medições são demoradas e onerosas, devido à quantidade de tempo envolvido na sua obtenção e na quantidade e qualidade do corpo avaliadores envolvidos nestas medições. Não se deve esquecer do preço dos instrumentos de medição, que na sua grande maioria são importados. Portanto, medições e mapas de ruído, quando forem disponíveis para uma mesma análise, ajudarão aos avaliadores e gestores urbanos e ambientais observar se as medições foram bem feitas, assim como se os mapas foram bem calculados. Esta questão é mostrada acima, quando se percebe que os dados medidos caem dentro da faixa de valores calculados pelos mapas. Portanto, a qualidade das medições caminha junto com a qualidade dos mapas de ruído e vice-versa.

\section{AGRADECIMENTOS}

Os autores gostariam de agradecer ao CNPq e ao DAAD (Deutscher Akademischer Austauschdienst) Serviço Alemão de Intercâmbio Acadêmico, por fornecerem os meios financeiros para a compra do analisador sonoro utilizado nas medições acústicas presentes neste trabalho. Os autores gostariam, também de externar o seu agradecimento, aos avaliadores que ajudaram a melhorar a qualidade do presente artigo. 


\section{REFERÊNCIAS BIBLIOGRÁFICAS}

ASTOLFI, A.; PELLEREY, F. Subjective and objective assessment of acoustical and overall environmental quality in secondary school classrooms. Journal of the Acoustics Society of America, v. 123, n 1, p. $16-173,2008$.

ASENSIO, C., RECUERO, M. \& MARIANO, R. Noise Mapping. Applied Acoustics, v. 72 (8), pgs. $477-478,2011$.

BUNN, F. \& ZANNIN, P.H.T. Assessment of railway noise in an urban setting. Apllied Acoustics, v. 104. pgs., 16 - 23, 2016.

CASAS, W. J. P.; CORDEIRO, E. P.; MELLO, T. C. \& ZANNIN, P. H. T. Noise mapping as a tool for controlling industrial noise pollution. Journal of Scientific \& Industrial Research, 73, 262-266, 2014.

DIESENDORF, M. Urban Transportation in the $21^{\text {st }}$ Century. Environmental Science and Policy; v. 3, pgs: 11-13, 2004.

FUNDACENTRO. NHO-01: Norma de Higiene Ocupacional, avaliação da exposição ocupacional ao ruído. São Paulo, 1999.

FIORINI, A. C. Impacto Ambiental e Ocupacional na Audição de Trabalhadores. In: FERNANDES, F. D. M.; MENDES, B. C. A.; NAVAS, A. L. P. G. P. (Orgs). Tratado de Fonoaudiologia. 2ª ed. São Paulo: Editora Roca, 2009.

FIEDLER, P.E.K., ZANNIN, P.H.T. Evaluation of noise pollution in urban traffic hubs - Noise maps and measurements. Environmental Impact Assessment Review, v. 51, pgs: 1-9, 2015. 
RUÍDO OCUPACIONAL EM ESTAÇÕES DE ÔNIBUS - ESTUDO DE CASO EM ESTAÇÕES TUBO - CURITIBA - PARANÁ - BRASIL

HÉTU, R.; TRUCHON-GAGNON, C.; BILODEAU, A. Problems of noise in school settings: A review of literature and the results of an exploratory study. J. Speech Lang. Path. Audiol., v. 14, p. 31 - 39, 1990.

HERBST, K. Brazil's model city. Planning, v. 58 (9), pgs: 24-27, 1992.

HAGEN, M.; HUBER, L.; KAHLERT, J. Acoustic School Desing. International Forum Acusticum, Sevilha, Proceedings, CD-ROM, Sevilha, 2002.

FIEDLER, P.E..K.; ZANNIN, P.H.T. Environmental Impact Assessment Review, v. 51, 1-9, 2015.

GOSWAMI, S. A study on traffic noise of two campuses of University, Balasore, India. Journal of Environmentl Biology, v. 32, p. 105-109, 2011.

GUEDES, I. C. M.; BERTOLI, S. R.; ZANNIN, P. H. T. Influence of urban shapes on environmental noise: A case study in Aracajú - Brazil. Sci Total Environ, 412-413, 66-76, 2011.

ISO 1999 - INTERNATIONAL ORGANIZATION FOR STANDARDIZATION ACOUSTICS - DETERMINATION OF OCCUPATIONAL NOISE EXPOSURE AND ESTIMATION OF NOISE-INDUCED HEARING IMPAIRMENT. Switzerland, 1990.

KENNEDY, M.; HODGSON, M.; EDGETT, D.; LAMB, N.; REMPEL, R. Subjective assessment of listening environments in university classrooms: Perceptions of students. Journal of the Acoustical Society of America, v. 119, n 1, p. 299-309, 2006.

KING, E. A.; RICE, H. J. The development of a practical framework for strategic noise mapping. Appl Acoust v. 70, pgs. 1116-27, 2009. 
PMC - LEI 10625 - PREFEITURA MUNICIPAL DE CURITIBA - Secretaria Municipal do Meio Ambiente - SMMA (Brasil). Lei Municipal n.ำ10.625, de 19 de fevereiro de 2002: Dispõe sobre ruídos urbanos, proteção do bem estar e do sossego público e dá outras providências, 2002.

LEE, S., CHANG, S.I. \& PARK., Y. Utilizing noise mapping for environmental impact assessment in a downtown redevelopment area of Seoul, Korea", Applied Acoustics, v 69, pgs. 704-714, 2008.

LAW, C, LEE, C., LUI, A.S.,YEUNG, M.K. \& LAM, K. Advancement of threedimensional noise mapping in Hong Kong. Appl Acoust 2011, v. 72 (8), pgs, 534-43, 2011.

MASCHKE, C. Preventive Medical Limits for Chronic Traffic Noise Exposure. Acustica, v. 85, p:448, 1999.

NR - 15 - NORMA REGULAMENTADORA 15, Atividades e operações insalubres, Anexo 1, pg. 212. Segurança e Medicina do Trabalho, 68ª Edição, Ministério do Trabalho, Manuais de Legislação Atlas, São Paulo. 2011.

NUdelmanN, A. A. et. al. PAIR - Perda Auditiva Induzida pelo Ruído Volume II. Rio de Janeiro: Editora Revinter, 2001.

NR - 17 - NORMA REGULAMENTADORA 17, ERGONOMIA, pg. 307. Segurança e Medicina do Trabalho, 68a Edição, Ministério do Trabalho, Manuais de Legislação Atlas, São Paulo. 2011.

PINTO, F.A.C.; MARDONES, M.D.M. Noise mapping of densely populated neighborhoods - example of Copacabana Rio de Janeiro - Brazil. Environ Monit Assess 155:309-18, 2009. 
RUÍDO OCUPACIONAL EM ESTAÇÕES DE ÔNIBUS - ESTUDO DE CASO EM ESTAÇÕES TUBO - CURITIBA - PARANÁ - BRASIL

PORTELA, S., e ZANNIN, P. H. T. Analysis of factors that influence noise levels inside urban buses. Journal of Scientific \& Industrial Research, v. 69, pgs. 684-687, 2010.

PORTElA, B. S, QUEIROGA, M., CONSTANTINI, A. e ZANNIN, P. H. T. Annoyance evaluation and the effect of noise on the health of bus drivers. Noise \& Health, v. 15, pgs: 301-306, 2013.

SANTOS DE SOUZA, R. Entendendo a questão ambiental. EDUNISC, Santa Cruz do Sul, 2000.

SoundPlan - BRAUNSTEIN E BERNDT. Sound Plan User's Manual, p. 293, 1999.

SMITH, N., e HENSHER, D. The future of exclusive busways: the Brazilian experience. Transport Reviews; v. 18 (2), pgs: 131-152, 2006.

SANTOS, G. A. de OLIVEIRA. Avaliação da poluição sonora de tráfego nas proximidades de estações-tubo em diferentes áreas de zoneamento da cidade de Curitiba, 2015. Dissertação de Mestrado em Meio Ambiente Urbano e Industrial - MAUI, Departamento de Engenharia Química, Universidade Federal do Paraná, Curitiba.

THAKUR, G.S. A study of noise around an educational institutional area. J. Environ. Science Eng., v. 48, pgs. 35-38, 2006.

TSAY, K., LIN, M \& CHEN, Y. Noise mapping in urban environments: a

Taiwan study. Applied Acoustics, v 70(7), pgs. 964-972, (2009).

WORLD HEALTH ORGANIZATION - WHO (UK). Résumé d'Orientation des Directives de l'OMS Relatives au Bruit dans l'Environmental, 2003. 
RUÍDO OCUPACIONAL EM ESTAÇÕES DE ÔNIBUS - ESTUDO DE CASO EM ESTAÇÕES TUBO - CURITIBA - PARANÁ - BRASIL

WORLD HEALTH ORGANIZATION. Burden of disease from environmental noise. Copenhagen, 2011.

ZANNIN P. H.T., CALIXTO A, DINIZ F.B., FERREIRA J.A.C. A survey of urban noise annoyance in a large Brazilian city: the importance of a subjective analysis in conjunction with an objective analysis, 2003. Enviromental Impact Assessment Review; v23, pgs: 245-255, 2003.

ZANNIN, P. H. T. Occupational Noise in Urban Buses. International Journal of Industrial Ergonomics; v36, pg: 901-905, 2006.

ZANNIN, P.H.T.; MARCON, C. R. Objective and subjective evaluation of the acoustic comfort in classrooms. Applied Ergonomics, v. 38, p. 675- 68, 2007.

ZANNIN, P.H.T; ZWIRTES, D.P. Evaluation of the acoustic performance of classrooms in public schools. Applied Acoustics, v. 70, p. 626-635, 2009.

ZANNIN, P. H. T.; SANT'ANA, D. Q. Noise mapping at different stages of a freeway redevelopment project - A case study in Brazil. Applied Acoustics, 72, 479-486, 2011.

ZANNIN, P.H.T., ENGEL, M.S., FIEDLER, P.E.K. \& BUNN, F. Characterization of environmental noise based on noise measurements, noisemapping and interviews: A case study at a university campus in Brazil. Cities, v. 31, pgs. $317-327,2013$. 